\title{
Soziologische Theorie
}

\author{
Ute Volkmann
}

Online publiziert: 14. Juli 2020

(C) Der/die Autor(en) 2020

Schwinn, Thomas: Soziale Ungleichheit in differenzierten Ordnungen. Zur Wechselwirkung zweier Strukturprinzipien. Tübingen: Mohr Siebeck 2019. 275 Seiten. ISBN 978-3-16-155874-0. Preis: $€ 49,-$.

Mit dem vorliegenden Buch hat Thomas Schwinn erneut aus zehn bereits publizierten sowie zwei unveröffentlichten Aufsätzen einen Band zusammengestellt, um den state of the art von zwanzig Jahren eigener konzeptioneller Theoriearbeit zu dokumentieren, dieses Mal zur Verknüpfung von Differenzierungstheorie und ungleichheitstheoretischen Perspektiven. Damit legt Schwinn nach „Max Weber und die Systemtheorie“" nun auch zum zweiten seiner drei großen theoretischen Forschungsthemen eine Zwischenbilanz vor. Jeweils drei Aufsätze sind zu einem Themenkomplex gebündelt. Im ersten Block finden sich mit dem eröffnenden Artikel aus dem Jahr 1998 und zwei bis dato unveröffentlichten Manuskripten grundlegende Texte zur „Verhältnisbestimmung von funktionaler Differenzierung und sozialer Ungleichheit“" (S. 8). Die Aufsätze des zweiten Blocks diskutieren das theorieverknüpfende Potenzial der Begriffe Inklusion, Exklusion und Intersektionalität. Im dritten Block sind Texte gebündelt, die den Staat als durch ungleiche soziale Lagen und Interessen strukturierte und gesellschaftliche Verteilungsprozesse legitimierende Instanz in den Fokus rücken. Im letzten Block finden sich Auseinandersetzungen mit alternativen gesellschaftstheoretischen Angeboten, angefangen mit Adrian Itschert über Uwe Schimank bis zu Hartmut Esser.

Ich will im Folgenden nicht die einzelnen Beiträge je für sich würdigen, sondern nach der gesellschaftstheoretischen Essenz der hier vorgelegten Zusammenschau

U. Volkmann $(\bowtie)$

Institut für Soziologie, Universität Bremen

Mary-Somerville-Straße 9, 28359 Bremen, Deutschland

E-Mail: ute.volkmann@uni-bremen.de 
fragen. Die Einleitung bringt sowohl die „wissenschafts- und theoriegeschichtlich“ (S. 4) begründeten, wechselseitigen „Rezeptionssperren“ (S. 1) der beiden Gesellschaftstheorien als auch die daraus abgeleitete Programmatik der zu leistenden Theoriearbeit systematisch auf den Punkt. Schwinn identifiziert fünf empirisch mögliche Ausprägungen des Verhältnisses der beiden Strukturprinzipien, die vom Primat sozialer Ungleichheit über die sozialstrukturelle Brechung institutioneller Handlungslogiken, ein- oder wechselseitige Förderung oder Indifferenz bis zum Primat funktionaler Differenzierung reichen (S. 4-6). Um dieser „Bandbreite an Beziehungsmöglichkeiten“ und damit der „Varianz sozialer Ordnungsphänomene“ gerecht zu werden, ,[müsste] [e]ine Theorie [...] so angelegt sein, dass sie das gesamte Spektrum konzeptionell zu erfassen vermag“. Bisherige Gesellschaftstheorien, so Schwinn, leisten genau dies nicht, weswegen sie ,,aufgebrochen und neu rekonstruiert werden [müssen]“ (S. 7).

Wie sieht dieser derzeitige Stand der Schwinn'schen Rekonstruktionsarbeit aus? Es liegt nahe, hier vor allem die grundlegenden Texte des ersten Themenblocks in den Blick zu nehmen, allen voran die beiden als unveröffentlicht markierten Manuskripte. Bei ,Zum Theoriedefizit der Ungleichheitsforschung“ handelt es sich um eine Kompilation von Textabschnitten aus zwei früheren Buchpublikationen, die in ihrer Zusammenstellung die wechselseitige Bedingtheit von Differenzierungsprozessen und Ungleichheitsstrukturen nun sehr viel stärker als theoretisches Argument hervortreten lässt. ,Was leistet die Gesellschaftstheorie für die Ungleichheitsanalyse?" ist demgegenüber ein wirklich neuer Beitrag, in dem sich Schwinn nochmals mit Luhmann und dessen These vom für die moderne Gesellschaft konstitutiven Primat funktionaler Differenzierung auseinandersetzt. Erstmals und im Unterschied zu anderen Beiträgen des Bandes bezieht er hier die erst 2017 veröffentliche Gesellschaftstheorie des frühen Luhmann ein, mit dem Ergebnis, dessen Primatthese nun noch dezidierter zurückweisen und die prinzipielle Gleichrangigkeit der beiden gesellschaftlichen Strukturprinzipien analytisch fundieren zu können. Schwinn arbeitet sich somit auch in diesem Forschungsprogramm an Luhmann ab, mit dem Ziel, dessen konzeptionelle Schwächen zu überwinden - gerade, weil er an ihm als dem Differenzierungstheoretiker in der Linie Max Webers, Schwinns zweitem oder eigentlichem Gewährsmann, festhält.

Schwinns zentrale These ist, dass funktionale Differenzierung und soziale Ungleichheit Strukturen eigenen Rechts mit je eigenen „Strukturlogiken“ (S. 49) sind, die weder ,auseinander abgeleitet werden [können]“ (S. 71) noch losgelöst vom jeweils anderen Strukturprinzip erklärbar sind. Sie bilden „Kontexte füreinander, deren jeweilige strukturierende Wirkungen jedoch nicht genügen, um das andere eindeutig festzulegen“ (S. 71). Bezogen auf die Prägekraft sozialer Ungleichheit argumentiert Schwinn ausgehend von Weber (S. 42-46), dass die „Empfänglichkeit für und Beeinflussbarkeit [...] durch sozialstrukturelle bzw. Klasseninteressen“" (S. 7) je nach gesellschaftlicher Sphäre variiert. Dies betrifft aber genau genommen nur den Prozess der Ausdifferenzierung. Hinsichtlich herausgebildeter institutioneller Ordnungen argumentiert Schwinn auf der Linie von Luhmann, wenn er von der Unveränderbarkeit der Codes oder Leitwerte ausgeht - welche er allerdings im jüngsten seiner Beiträge in Anlehnung an Pierre Bourdieu in Frage stellt: „Eine Perspektive, die von Graden der Autonomie und Differenzierung der Bereiche ausgeht, hat 
den Vorteil, [...] die variierende Anfälligkeit der Institutionen für eine soziale Ungleichheitsstrukturierung konzeptionell und empirisch zum Gegenstand der Analyse machen zu können“ (S. 67).

Aus Sicht der Rezensentin wäre dieser Schritt für die weitere Ausarbeitung der Gesellschaftstheorie essenziell, um über die bisherige Konzeption hinaus zu kommen, die die Wirkmächtigkeit der Prägekräfte auf verschiedenen analytischen Ebenen verortet. Denn die Wirkungen funktionaler Differenzierung beziehen sich auf zumeist organisationale Positionen und auf Ressourcen, mithin auf das, was soziale Ungleichheit im Kern ausmacht: Die institutionellen Ordnungen, die das Individuum in seinem Lebenslauf diachron und synchron durchläuft, definieren die legitimen „Leistungskriterien“ (S. 91) für den Ressourcenerwerb. Die genuine Strukturlogik sozialer Ungleichheit kommt bislang demgegenüber erst in den ,nicht durch die Leitkriterien und Leistungserfordernisse der Ordnungen determiniert[en]“ (S. 38) Konflikt-, Monopolisierungs- und Schließungsprozessen zum Tragen. Bildete also bis dato letztlich die Differenzierungstheorie die Basis der Schwinn'schen Gesellschaftstheorie - jedenfalls ab dem Zeitpunkt, wo sich Sphären als eigenlogische gesellschaftliche Bereiche ausdifferenziert haben -, so deuten die aktuellsten Überlegungen diesbezüglich eine konzeptionelle Öffnung an.

Wenn Schwinn diesen Weg weiter beschreitet, liegt es aus Sicht der Rezensentin jedoch nahe, Bourdieu neben Weber und Luhmann als dritten Gewährsmann hinzuzuziehen. Luhmanns Differenzierungstheorie ist zugegebenermaßen analytisch in einigen Punkten präziser, etwa im Einbezug formaler Organisationen, aber Bourdieu betrachtet ausgehend von relativer Autonomie die Spielregeln und letztlich auch den nomos sozialer Felder als umkämpfte Sachverhalte. Und es sind diese, jeweils unter dem Einsatz von Kapital geführten Kämpfe um Definitionsmacht über die Logik, die Einsätze und die Regeln des Feldes, die auf der Ebene der Praktiken die Schnittstelle zwischen Differenzierungs- und Ungleichheitstheorie bilden. Auch die von Schwinn richtig konstatierten Defizite der Luhmann'schen Differenzierungstheorie, institutionelle Ordnungen weder als Arenen von ,Verteilungs- und Machtkämpfe[n]“ (S. 34) noch als hierarchisch strukturiert aufzufassen (S. 118), sprechen für Bourdieu als Gewährsmann. Dass sich darüber hinaus einige „Einsichten“ (S. 7) Schwinns relativ problemlos mit Bourdieu reformulieren lassen, wie Daniel Witte in seinem Beitrag auf dem Bochumer und Dortmunder DGS-Kongress 2012 gezeigt hat, ließe sich als Zusatzargument anführen.

Als Fazit lässt sich festhalten: Bezogen auf Schwinns state of the art der konzeptionellen Verknüpfung funktionaler Differenzierung und sozialer Ungleichheit bietet der Band zwangsläufig nur wenig Neues. Aber allein die Zusammenschau seiner gesellschaftstheoretischen Überlegungen über den Zeitraum von nunmehr zwanzig Jahren macht die Lektüre des Buches lohnend - trotz gewisser bei einem solchen Buchprojekt unvermeidbaren Redundanzen, denen auf der Habenseite jedoch ein Personen- sowie umfangreiches Sachregister gegenübersteht. Liest man die Texte kapitelweise, werden die analytischen Schwerpunktsetzungen und damit das Was der Schwinn'schen Theoriearbeit greifbar; liest man die Texte chronologisch, so bietet der Band daneben einen spannenden Einblick in die „Phasen“ der Schwinn'schen „Denkbewegung“ (S. 8), also in das Wie der Rekonstruktionsarbeit. Man darf ge- 
spannt sein auf das, was nun daraus folgt - gesellschaftstheoretisch und vielleicht endlich auch in der empirischen Forschung.

Funding Open Access funding provided by Projekt DEAL.

Open Access Dieser Artikel wird unter der Creative Commons Namensnennung 4.0 International Lizenz veröffentlicht, welche die Nutzung, Vervielfältigung, Bearbeitung, Verbreitung und Wiedergabe in jeglichem Medium und Format erlaubt, sofern Sie den/die ursprünglichen Autor(en) und die Quelle ordnungsgemäß nennen, einen Link zur Creative Commons Lizenz beifügen und angeben, ob Änderungen vorgenommen wurden.

Die in diesem Artikel enthaltenen Bilder und sonstiges Drittmaterial unterliegen ebenfalls der genannten Creative Commons Lizenz, sofern sich aus der Abbildungslegende nichts anderes ergibt. Sofern das betreffende Material nicht unter der genannten Creative Commons Lizenz steht und die betreffende Handlung nicht nach gesetzlichen Vorschriften erlaubt ist, ist für die oben aufgeführten Weiterverwendungen des Materials die Einwilligung des jeweiligen Rechteinhabers einzuholen.

Weitere Details zur Lizenz entnehmen Sie bitte der Lizenzinformation auf http://creativecommons.org/ licenses/by/4.0/deed.de.

Ute Volkmann Dr., Mitarbeiterin am Institut für Soziologie und am SOCIUM der Universität Bremen. Forschungsschwerpunkte: soziologische Gesellschaftstheorien, soziologische Gegenwartsdiagnosen, Wirtschaftssoziologie und Mediensoziologie. Aktuelle Publikationen: Gesellschaftstheorie und Gegenwartsdiagnosen. Verhältnisbestimmungen und Themenpanorama. In: Gegenwartsdiagnosen: Kulturelle Formen gesellschaftlicher Selbstproblematisierung in der Moderne. Bielefeld 2019 (mit U. Schimank, Hrsg. T. Alkemeyer, N. Buschmann, T. Etzemüller); Gesellschaftliche Ökonomisierung und die Gegenkräfte: ein differenzierungstheoretischer Bezugsrahmen. In: Ökonomisierung. Debatten und Praktiken in der Zeitgeschichte. Göttingen 2019 (Hrsg. Rüdiger Graf). 